\title{
Biochar Alters the Root Systems of Large Crabgrass
}

\author{
Karen Mitchell, Elizabeth French, Janna Beckerman, and \\ Anjali Iyer-Pascuzzi
}

Department of Botany and Plant Pathology, Purdue University, West Lafayette, IN 47907

\section{Jeff Volenec \\ Department of Agronomy, Purdue University, West Lafayette, IN 47907}

\section{Kevin Gibson ${ }^{1}$}

Department of Botany and Plant Pathology, Purdue University, West Lafayette, IN 47907

Additional index words. black carbon, rhizotron, rhizobox, root system architecture

\begin{abstract}
Soil incorporation of biochar appears to increase plant growth in some environments. However, the effect of biochar on root system architecture (RSA) or on weeds is not well understood. Our objective was to examine the effect of biochar on the growth and RSA of large crabgrass (Digitaria sanguinalis L. Scop.), a common and problematic weed. Plants were grown in rhizoboxes filled with field soil \pm either a lownutrient biochar (LNB) or a high-nutrient biochar (HNB). Rhizoboxes were either filled uniformly with field soil \pm biochar (solid) or with + biochar and - biochar-amended field soil so that each occupied half of the rhizobox (split). Large crabgrass biomass and RSA were affected by biochar type in the solid design rhizoboxes and large crabgrass roots proliferated in biochar-amended soil in the split rhizoboxes, regardless of biochar type. This study provides evidence that plant roots can detect and grow toward biochar and suggests that the addition of biochar to soils may increase the ability of large crabgrass to spread vegetatively.
\end{abstract}

Biochar is a carbon-rich product formed through the pyrolysis of organic matter. A meta-analysis of 371 independent studies from 114 published manuscripts determined that the addition of biochar to soils resulted in increased crop yields, soil microbial biomass, rhizobia nodulation, soil phosphorus $(\mathrm{P})$, soil potassium $(\mathrm{K})$, and total nitrogen $(\mathrm{N})$ and carbon (C) (Biederman and Harpole, 2013). Similarly, a meta-analysis conducted by Jeffery et al. (2011) reported that biochar additions to the soil resulted in an average increase of $10 \%$ in crop productivity. Biochar has been proposed as a soil amendment to sequester carbon and improve soil properties and crop yields. However, biochar may increase weed growth in addition to crop productivity. Although weeds are known to reduce crop yields, there is little published research on the effect of biochar on weeds (Major et al., 2005; Quilliam et al., 2012; Smith and Cox, 2014; Soni et al., 2014). Smith and Cox (2014) showed a neutral effect of biochar on parasitic yellow rattle (Rhinanthus minor) abundance. Biochar did not increase the biomass of palmer amaranth (Amaranthus palmeri), sicklepod (Senna obtusifolia), and southern crabgrass (Digitaria ciliaris), although it did reduce germination in palmer

Received for publication 7 Nov. 2017. Accepted for publication 6 Dec. 2017.

${ }^{1}$ Corresponding author. E-mail: kgibson@purdue. edu. amaranth (Soni et al., 2014). Quilliam et al. (2012) reported no effect of biochar on weeds 3 years after incorporation at 25 or $50 \mathrm{t}$ biochar/ha in a sandy clay loam soil. However, weed emergence was reduced when biochar was reapplied. The authors were unable to explain the reduction in weed emergence but suggested that increased soil microbial activity might play a role (Quilliam et al., 2012). Biochar applied alone did not increase weed cover but biochar plus an inorganic fertilizer increased weed cover more than the inorganic fertilizer alone in low fertility, highly acidic soils of the central Brazilian Amazon (Major et al., 2005).

Biederman and Harpole (2013) determined that annual plants (no response was detected for perennial plants) responded to biochar with increased belowground growth but they did not detect an effect of biochar on biomass partitioning. They therefore suggested that, in annuals, biochar increases both above- and belowground growth. However, this conclusion was based on a small number of studies $(n=10)$ (Biederman and Harpole, 2013), and the effect of biochar on RSA, which is the spatial arrangement or topology of plant roots, has been examined in only a few studies (Abiven et al., 2015; Olmo et al., 2016; Prendergast-Miller et al., 2011). Khan and Shea (2012) concluded that biochar can increase turf root growth in the Jandakot sands of West Australia. We are unaware of any published studies in which the effect of biochar on weed RSA was examined.
The objective of this study was to examine the effects of two types of biochar on root growth and RSA of large crabgrass (D. sanguinalis L. Scop.) using a rhizobox mesocosm. Large crabgrass is a common and problematic annual weed in turfgrass and agricultural settings (Mitich, 1988; Turner et al., 2012). Large crabgrass has fibrous roots, an often prostrate growth habit, and can produce adventitious roots at stem nodes and form thick mats (Mitich, 1988). Crabgrass appears to have relatively high $\mathrm{P}$ and $\mathrm{K}$ requirements, and reduction in soil $\mathrm{P}$ and $\mathrm{K}$ availability can significantly reduce large crabgrass growth (Hoveland et al., 1976). We hypothesized that large crabgrass roots would proliferate in soil amended with biochar, particularly in soil amended with the high-nutrient biochar.

\section{Materials and Methods}

A sandy loam field soil, Desker series (coarse-loamy, mixed, superactive, and mesic Mollic Hapludalfs), was collected in June 2013 from the top $10 \mathrm{~cm}$ of a conventional agricultural field located at Throckmorton Purdue Agricultural Center (lat. $40^{\circ} 17^{\prime} 42.0^{\prime \prime} \mathrm{N}$, long. $\left.86^{\circ} 54^{\prime} 33.8^{\prime \prime} \mathrm{W}\right)$. Biochar produced at the same temperature $\left(450{ }^{\circ} \mathrm{C}\right)$ but from two different feedstocks was used in this experiment. Organic matter, $\mathrm{pH}$, cation exchange capacity (CEC), and extractable Bray 2-phosphorus, $\mathrm{K}$, magnesium $(\mathrm{Mg})$, and calcium $(\mathrm{Ca})$ were determined by a commercial laboratory (A\&L Great Lakes Laboratories, Fort Wayne, IN). Total $\mathrm{C}$ and $\mathrm{N}$ were determined for the affected soils in quintuplicate by element analyzer (Thermo Scientific FlashEA 1112 series; Thermo Fisher Scientific, Waltham, MA). The HNB was produced from a mixture of loblolly pine (Pinus taeda) and switchgrass (Panicum virgatum) by a commercial producer (Eprida, Inc., Marietta, GA) and had greater CEC, total C and $\mathrm{N}, \mathrm{K}$, $\mathrm{Mg}$, and $\mathrm{Ca}$ and extractable Bray phosphorus than the LNB (Table 1). The LNB was produced from a mixture of fir, pine, and spruce by a commercial producer (Diacarbon Energy Inc., Burnaby, BC, Canada) and had greater $\mathrm{pH}$ and $\mathrm{C}: \mathrm{N}$ ratios than the HNB (Table 1). After passing the soil and biochar separately through a 4-mm sieve, the field soil was amended with one of the two types of biochar at a rate of $2 \%$ of the soil dry weight (DW) $\left(\approx 20 \mathrm{t} \cdot \mathrm{ha}^{-1}\right)$ and thoroughly mixed in a $50-\mathrm{L}$ electric concrete mixer for $2 \mathrm{~h}$.

Large crabgrass (Azlin Seed Service, Leland, MS) was grown in rhizoboxes to examine the effect of two types of biochar on root growth and RSA. Rhizoboxes were constructed using two transparent acrylic sheets and three wooden spacers held together with medium binder clips to make interior dimensions of $24 \times 20 \times 0.5 \mathrm{~cm}$ $(H \times W \times D)$. Each rhizobox was filled with soil ( \pm biochar) to create a $0.5-\mathrm{cm}$ layer of soil between the acrylic sheets. Two soil patterns were used: solid and split (Fig. 1). The solid pattern consisted of unamended or amended soil positioned uniformly throughout a rhizobox. 


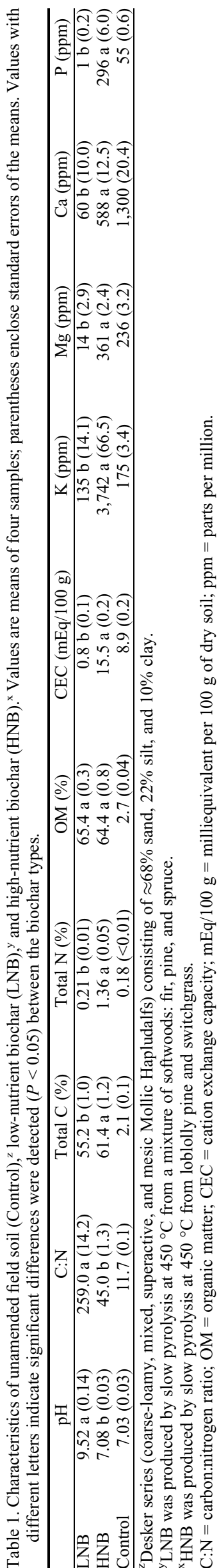

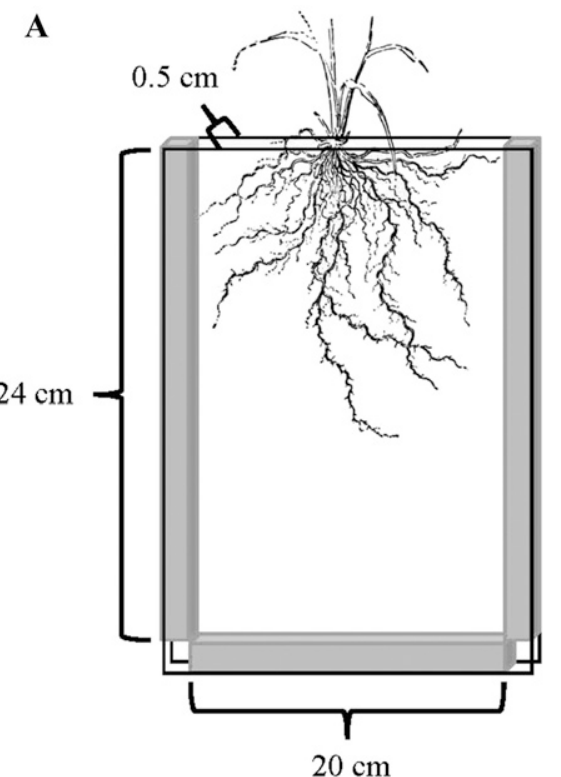

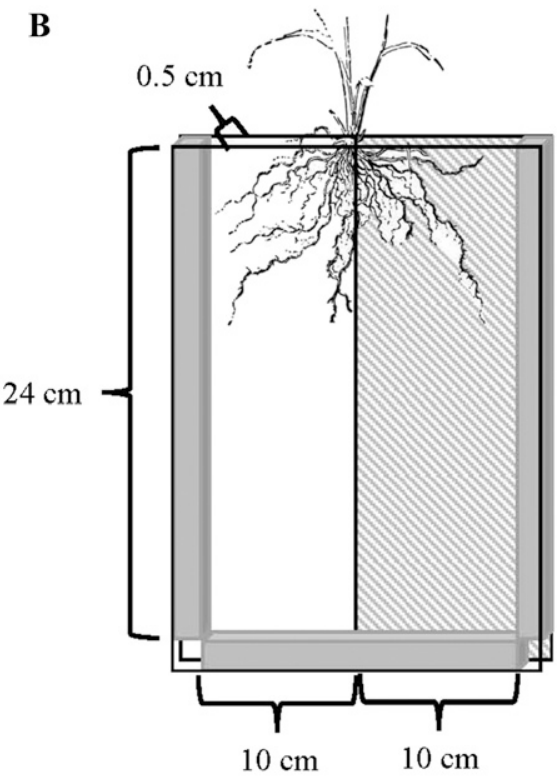

Fig. 1. A diagram of rhizobox construction and soil patterns. Rhizoboxes were made from two transparent acrylic sheets $(0.3 \mathrm{~cm}$ thick) with wood spacers $(0.5 \mathrm{~cm}$ thick $)$ in between the acrylic sheets on both sides and bottom (shown in pale grey) and held together with medium binder clips. The solid-pattern (A) is uniformly filled with field soil ( $\pm 2 \%$ biochar). The split pattern $(\mathbf{B})$ is filled with unamended field soil and field soil amended with $2 \%$ biochar, each occupying half of the rhizobox vertically.

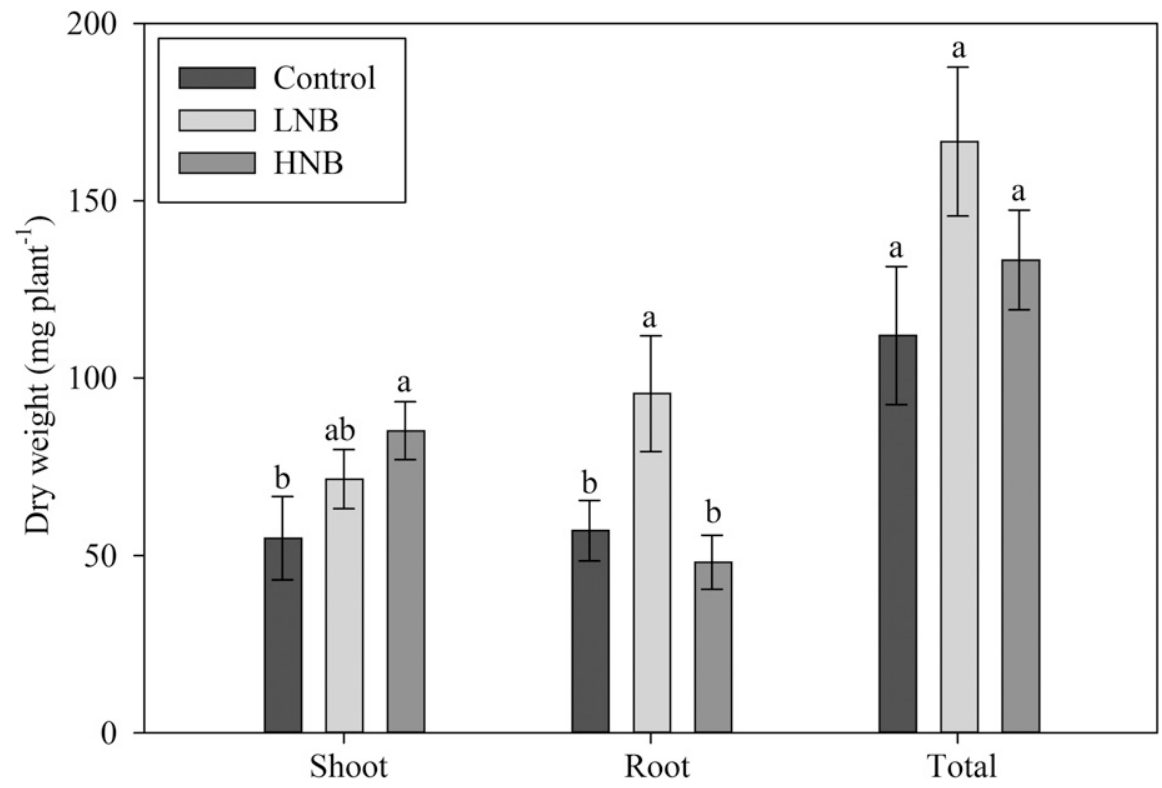

Fig. 2. Shoot, root, and total plant dry weight of large crabgrass grown in solid-pattern rhizobox with unamended field soil (Control), field soil with 2\% low-nutrient biochar (LNB), or field soil with $2 \%$ high-nutrient biochar (HNB). Columns represent means; error bars represent standard error of the mean. Means with different letters indicate significant statistical difference $(P<0.05)$.

The split pattern consisted of unamended and amended soil, each occupying half of a rhizobox vertically. The split pattern design was used to determine if large crabgrass would preferentially grow toward the control or amended soils when presented with both options. Large crabgrass seeds were germinated on moist filter paper, and a single seedling with a $20-\mathrm{mm}$ radicle was placed in the top center of each rhizobox. Aluminum foil was wrapped around the rhizoboxes to exclude light. The rhizoboxes were completely randomized in two growth chambers (Conviron PGR15, Winnipeg, Canada) with four replications of each treatment in each growth chamber. The rhizoboxes were kept at a $65^{\circ}$ angle to force roots to grow against the acrylic sheet (Brennan et al., 2014). Plants were grown for $38 \mathrm{~d}$ at $28 / 18{ }^{\circ} \mathrm{C}$ day/night temperatures, respectively, with a 15 -h photoperiod (9 h night) to imitate average Indiana summer conditions. Photosynthetically active radiation $(P A R)$ in the growth chambers was $500 \mu \mathrm{mol} \cdot \mathrm{m}^{-2} \cdot \mathrm{s}^{-1}$ (AccuPAR LP-80 PAR/LAI 


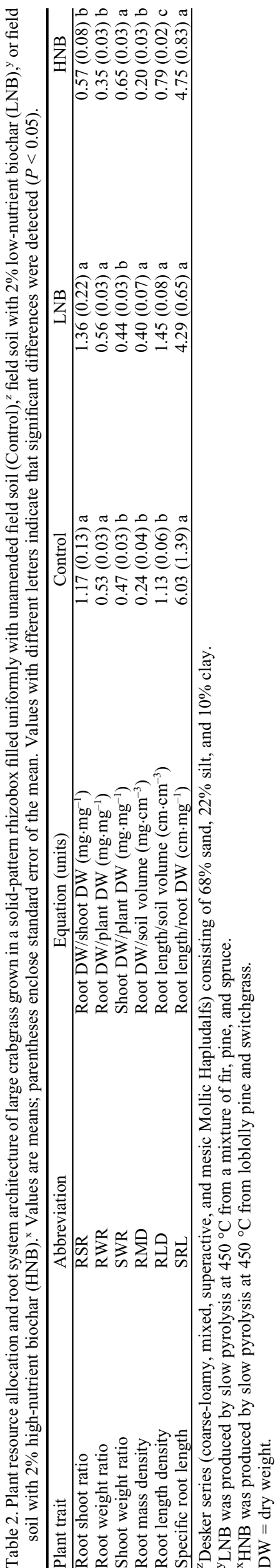

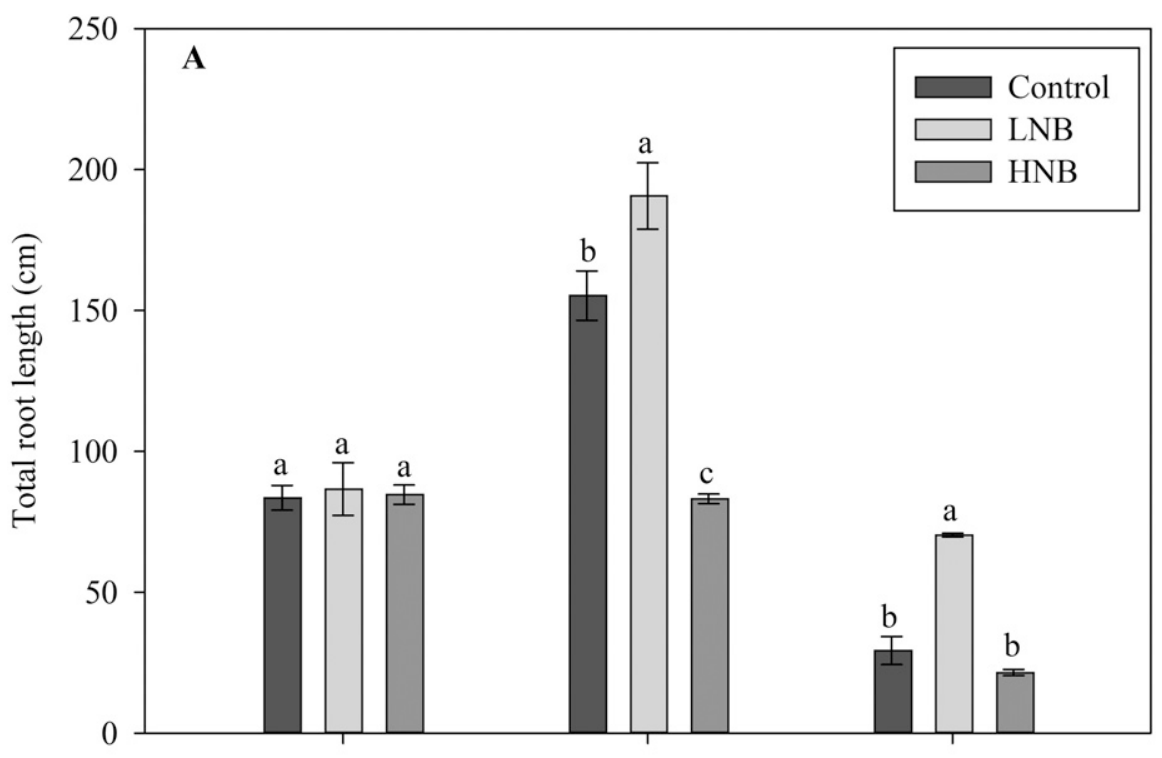

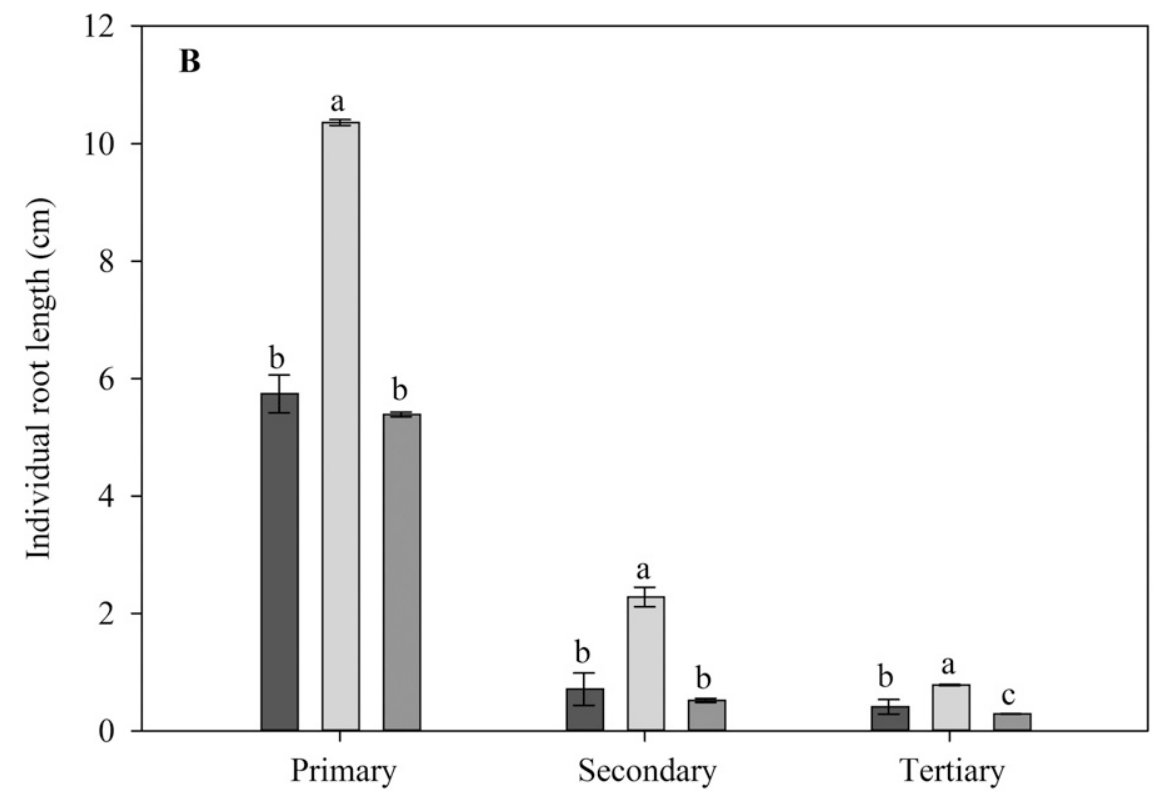

Fig. 3. Root length of primary, secondary, and tertiary roots of large crabgrass grown in solid-pattern rhizobox with unamended field soil (Control), field soil with $2 \%$ low-nutrient biochar (LNB), or field soil with $2 \%$ high-nutrient biochar (HNB). Total root length (A) represents the sum of all roots within a root order (primary, secondary, or tertiary). Individual root length $(\mathbf{B})$ represents the average length of a single root within a root order (primary, secondary, or tertiary). Columns represent means; error bars represent standard error of the mean. Within a root order, means with different letters indicate significant statistical difference $(P<0.05)$.

Ceptometer; Decagon Devices Inc., Pullman, WA). The plants were watered daily with $20 \mathrm{~mL}$ of distilled (DI) water.

At $38 \mathrm{~d}$ after transplant, rhizoboxes were scanned with an Epson Perfection V37 desktop scanner at $600 \mathrm{dpi}$. Height and number of tillers were recorded before the plants were harvested by cutting the stem at the soil surface. Leaves and stems were placed separately in paper bags and dried at $60{ }^{\circ} \mathrm{C}$ to a constant weight. Roots were removed from the soil using dissecting forceps for both rhizobox types. However, the root systems in the splitpattern rhizoboxes were cut down the center with a straight blade before the roots were removed. Roots were gently washed in DI water and dried at $60{ }^{\circ} \mathrm{C}$ to a constant weight.
Root images were analyzed using ImageJ 1.41o (Schneider et al., 2012) with SmartRoot (Lobet et al., 2011) plug-in for root length, root diameter, number of roots within each root order, and insertion angle, which is the angle at which new branches emerge from "parent" roots. The roots were not completely visible at the top of the rhizobox; therefore, root analysis was started $2 \mathrm{~cm}$ from the soil surface. Large crabgrass has a complex fibrous root system with multiple primary or seminal roots. Primary roots were identified in the root images and visually confirmed at harvest. Laterals branching from the primary roots are referred to as secondary roots, laterals branching from the secondary roots are referred to as tertiary roots, and so forth. 
Three estimates of root length were calculated: total root system length, total root length, and individual root length. Total root system length is the sum of all roots in the entire root system, disregarding root order (primary, secondary, and tertiary). Total root length is the sum of all roots within a root order. Individual root length is the average length of a single root within a root order. Several plant resource allocation and RSA ratios were calculated: root $\mathrm{DW} /$ shoot $\mathrm{DW}$ (root shoot ratio, RSR); root DW/total DW (root weight ratio, RWR); shoot DW/total DW (shoot weight ratio, SWR); root DW/soil volume (root mass density, RMD); root system length/soil volume (root length density, RLD); and root system length/root DW (specific root length, SRL).

Statistical analyses of the results were completed using SAS 9.3 (SAS Institute Inc., Cary, NC). Data were tested for normality and homogeneity of variances, and transformations were not necessary. Analysis of variance was used to determine differences among treatments. No biochar by growth chamber interactions were detected; therefore, data from both growth chambers were pooled (each treatment therefore had eight replications). Each soil pattern was analyzed separately. Data from the split-pattern rhizoboxes were analyzed to allow comparisons of growth within a rhizobox, i.e., between amended and unamended halves and comparisons between rhizoboxes containing the two biochars. The latter analyses were conducted by comparing growth in the unamended LNB half to growth in the unamended HNB half and by comparing growth in the LNB-amended half to growth in the HNB-amended half. Least-square means tests were completed for mean comparisons of all dependent variables.

\section{Results}

Solid-pattern rhizoboxes. Biochar did not affect large crabgrass total DW and differences in shoot DW were only detected between the HNB and control treatments (Fig. 2). Root DW was greater for plants grown with LNB than for control plants or plants grown with HNB (Fig. 2). More tillers were produced by plants grown in the $\mathrm{HNB}(2.8$ tillers $\pm 0.3 \mathrm{SE})$ and LNB (2.3 tillers $\pm 0.2 \mathrm{SE})$ treatments than in the control $(1.3 \pm 0.2 \mathrm{SE})$ treatment. Plants grown in the control treatment partitioned more biomass to roots and had greater RLD than plants grown in the HNB treatment (Table 2). Differences in biomass partitioning and RSA between the control and LNB treatments were only detected for RMD and RLD (Table 2). Biochar did not affect the total length of primary roots (Fig. 3A), but the individual length of primary roots was greater for plants grown in LNB soil than for plants grown in the HNB or the control soils (Fig. 3B). Total and individual root length of secondary and tertiary roots was greater for plants grown in LNB soil than for plants grown in the HNB or control treatments. No differences were detected in the individual root length of primary or secondary roots between the HNB and control treatments. However, the individual root length of tertiary roots was greater in the control than in the HNB treatment (Fig. 3A). Root diameters of secondary and tertiary roots were greater for both biochar types than for the control (Fig. 4). Primary root diameters were greater for plants grown in the
LNB than in the HNB or control treatments. Insertion angles were greater for the control treatment than for the LNB treatment for secondary and quaternary roots and for the HNB treatment, regardless of root order (Fig. 5).

Split-pattern rhizoboxes. Shoot DWs for the split-pattern rhizoboxes were $64.9 \mathrm{mg} /$ plant \pm 8.9 SE and $72.3 \mathrm{mg} /$ plant $\pm 7.8 \mathrm{SE}$ for large crabgrass grown with LNB and HNB,

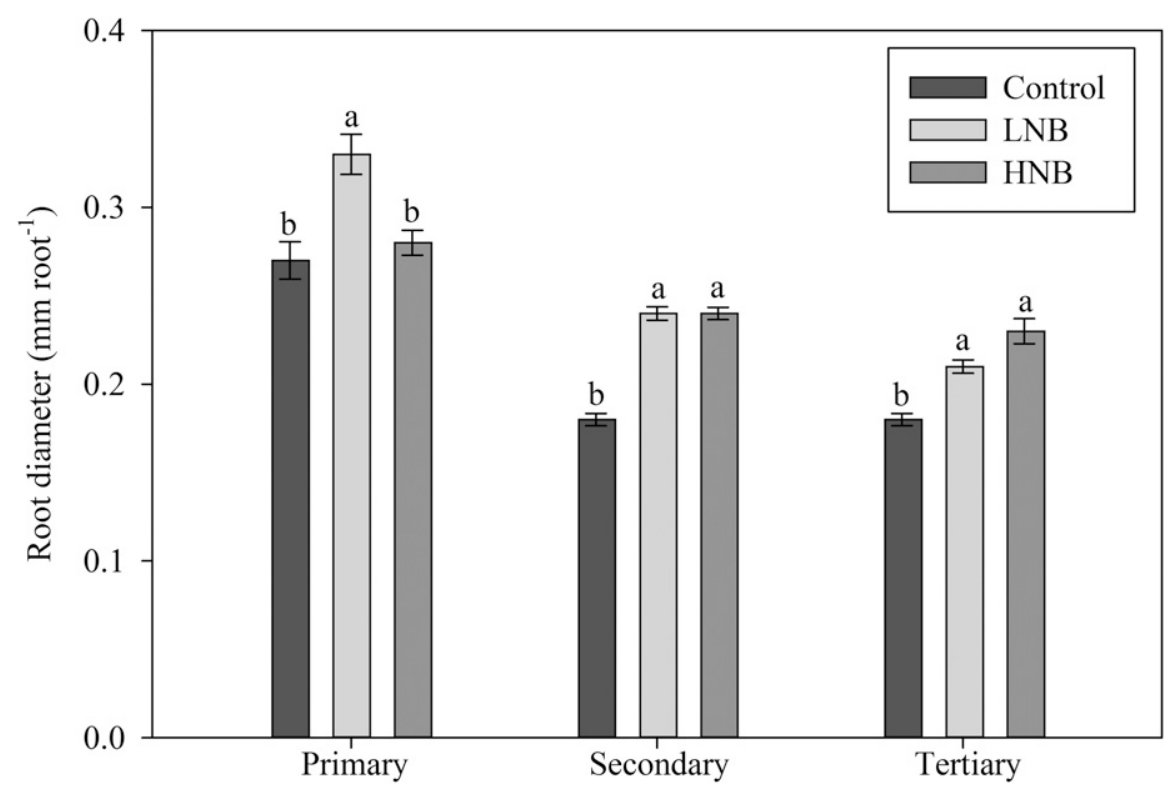

Fig. 4. Root diameter of primary, secondary, and tertiary roots of large crabgrass grown in solid-pattern rhizobox with unamended field soil (Control), field soil with $2 \%$ low-nutrient biochar (LNB), or field soil with $2 \%$ high-nutrient biochar (HNB). Columns represent means; error bars represent standard error of the mean. Within a root order, means with different letters indicate significant statistical difference $(P<0.05)$.

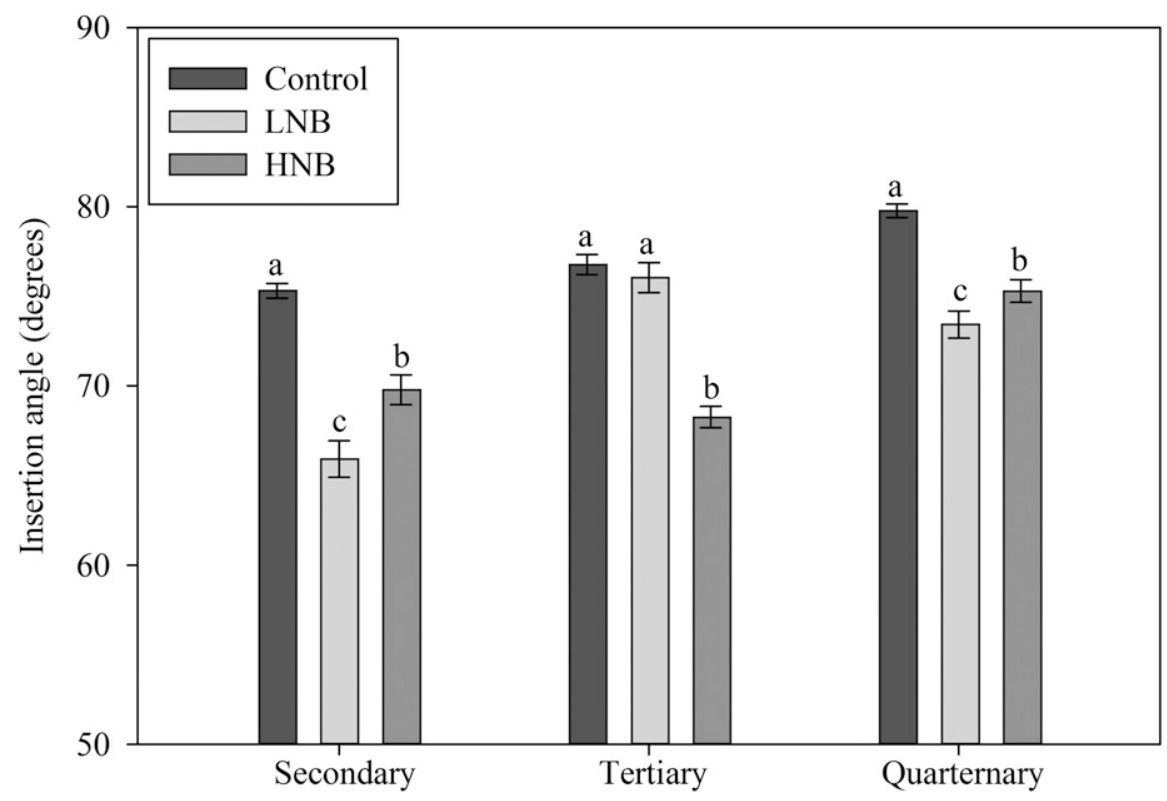

Fig. 5. Branch insertion angle of secondary, tertiary, and quaternary roots of large crabgrass grown in solidpattern rhizobox with unamended field soil (Control), field soil with $2 \%$ low-nutrient biochar (LNB), or field soil with $2 \%$ high-nutrient biochar (HNB). Columns represent means; error bars represent standard error of the mean. Within a root order, means with different letters indicate significant statistical difference $(P<0.05)$. 
respectively. Root DW and total root system length were greater in the half of the rhizobox amended with biochar regardless of the type of biochar (Fig. 6). Individual and total root lengths were greater in the LNB soil than in the HNB soil (Fig. 7). Biochar did not affect RMD in the split-pattern rhizobox (data not shown) but RLD and SRL were greater in the LNB soil $(1.2 \pm 0.12 \mathrm{SE}$ and $10.8 \pm 1.4 \mathrm{SE}$, respectively) than in the HNB soil $(0.5 \pm 0.1$ SE and $4.8 \pm 0.7 \mathrm{sE}$, respectively). No differences were detected between the two control halves for any dependent variable (data not shown).

\section{Discussion}

The rhizobox experiments suggest that large crabgrass biomass partitioning and RSA
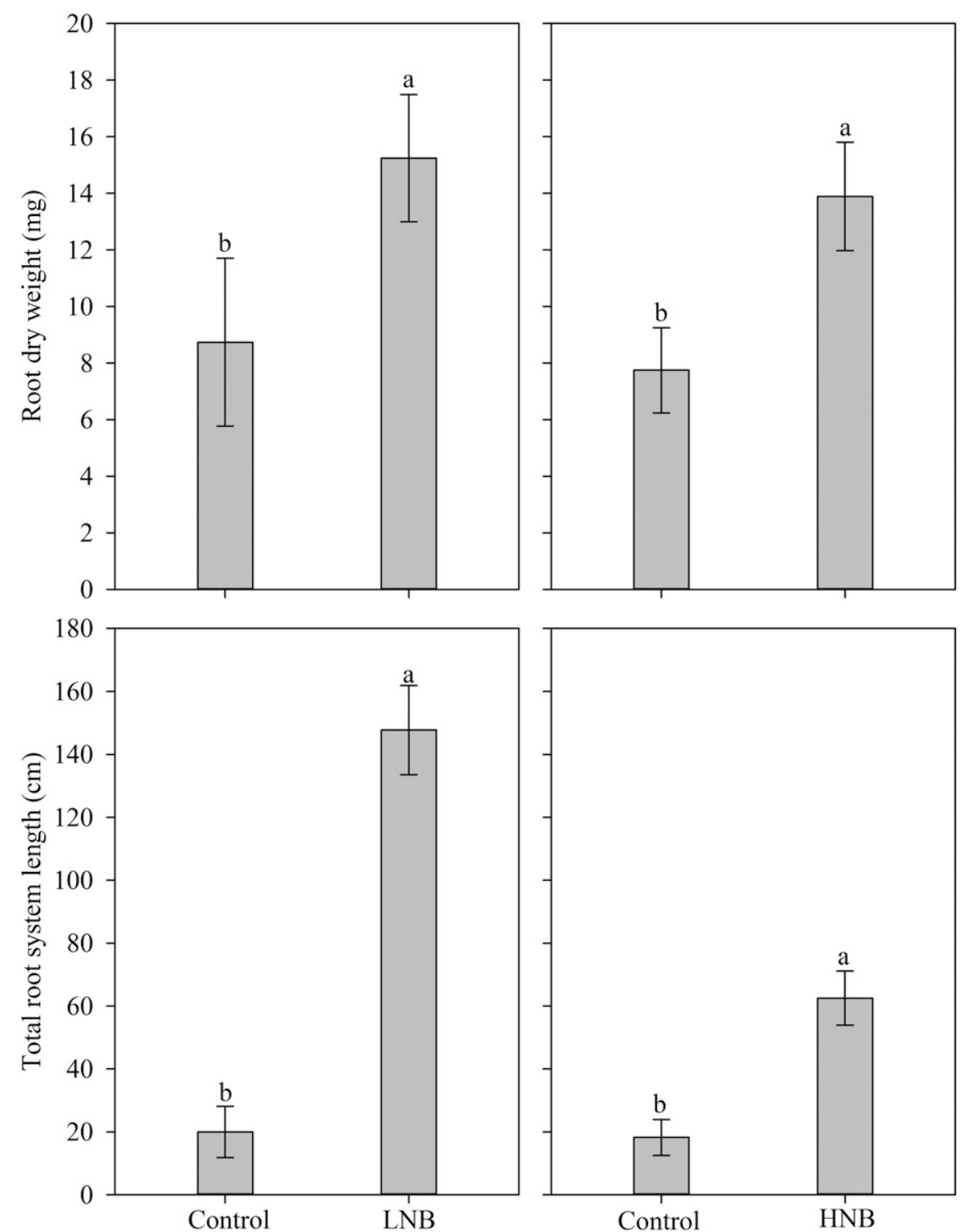

Fig. 6. Root dry weight and total root system length of large crabgrass grown in split-pattern rhizobox. Large crabgrass seedlings were placed in the center of the rhizobox with unamended field soil (Control) and field soil amended with either 2\% low-nutrient biochar (LNB) or high-nutrient biochar (HNB), each occupying half of the rhizobox vertically. Columns represent means; error bars represent standard error of the mean. Columns with different letters indicate significant statistical difference $(P<0.05)$. levels in a localized section of the root zone resulted in the proliferation of barley roots in the area with a high nutrient level. Biochar in the split-pattern rhizoboxes may have acted as a high-nutrient "patch" toward which the crabgrass roots grew.

The effect of biochar on large crabgrass depended on type of biochar and may reflect different foraging strategies for plants responding to HNB and LNB. The solidpattern results suggest a fertilizer effect in which the addition of HNB to the soil reduced the need for large crabgrass to invest in roots to take up nutrients. Olmo et al. (2016) observed similar results in their study examining RSA traits in wheat grown in pots amended with different levels of two biochars and different levels of fertilization. The biochar containing high levels of available $\mathrm{P}$ and $\mathrm{K}$ decreased RLD, wheat root biomass, and RWR, paralleling the effects of HNB on crabgrass root growth in our study. However, plants grown in the LNB soil had longer and thicker primary roots in the solid-pattern rhizoboxes than plants grown in the HNB and control soils. Lynch (2012) suggested that in an environment with low $\mathrm{N}$ availability, roots tend to have smaller insertion angles and develop proliferative and deep growth to forage for $\mathrm{N}$, a mobile nutrient generally found deeper in the soil. Thus, it is possible that the differential response of large crabgrass roots to the biochar types may reflect differences in nutrient availability associated with the LNB and HNB biochar. However, biochar has multiple effects on soils and it is possible that large crabgrass responded to factors in addition to or instead of nutrient availability. For example, biochar may contain other signaling molecules that alter RSA, such as the plant hormone ethylene (Spokas et al., 2010). Additional research is needed to more fully understand the effect of biochar on root growth and architecture. While changes to soil nutrient are the most obvious possibilities, other factors, such as the presence of chemicals that affect root growth or biochar-mediated effects on soil physical characteristics or the rhizosphere microbiome, should also be investigated.

This is the first detailed characterization of large crabgrass root growth and RSA and, to our knowledge, the first study to demonstrate preferential growth of roots into biochar-amended soil over unamended soil. This study suggests that the roots of large crabgrass, a globally important weed in turfgrass and agricultural systems, will preferentially spread into soil enriched with biochar. Furthermore, the addition of biochar to soils has the potential to increase large crabgrass tiller production and to increase the ability of large crabgrass to produce longer primary roots to forage for nutrients. Although biochar soil additions may be useful for improving nutrient uptake and yields in crop and turf plants, these benefits may be mitigated if biochar additions also result in unintended effects such as increased weed growth. 

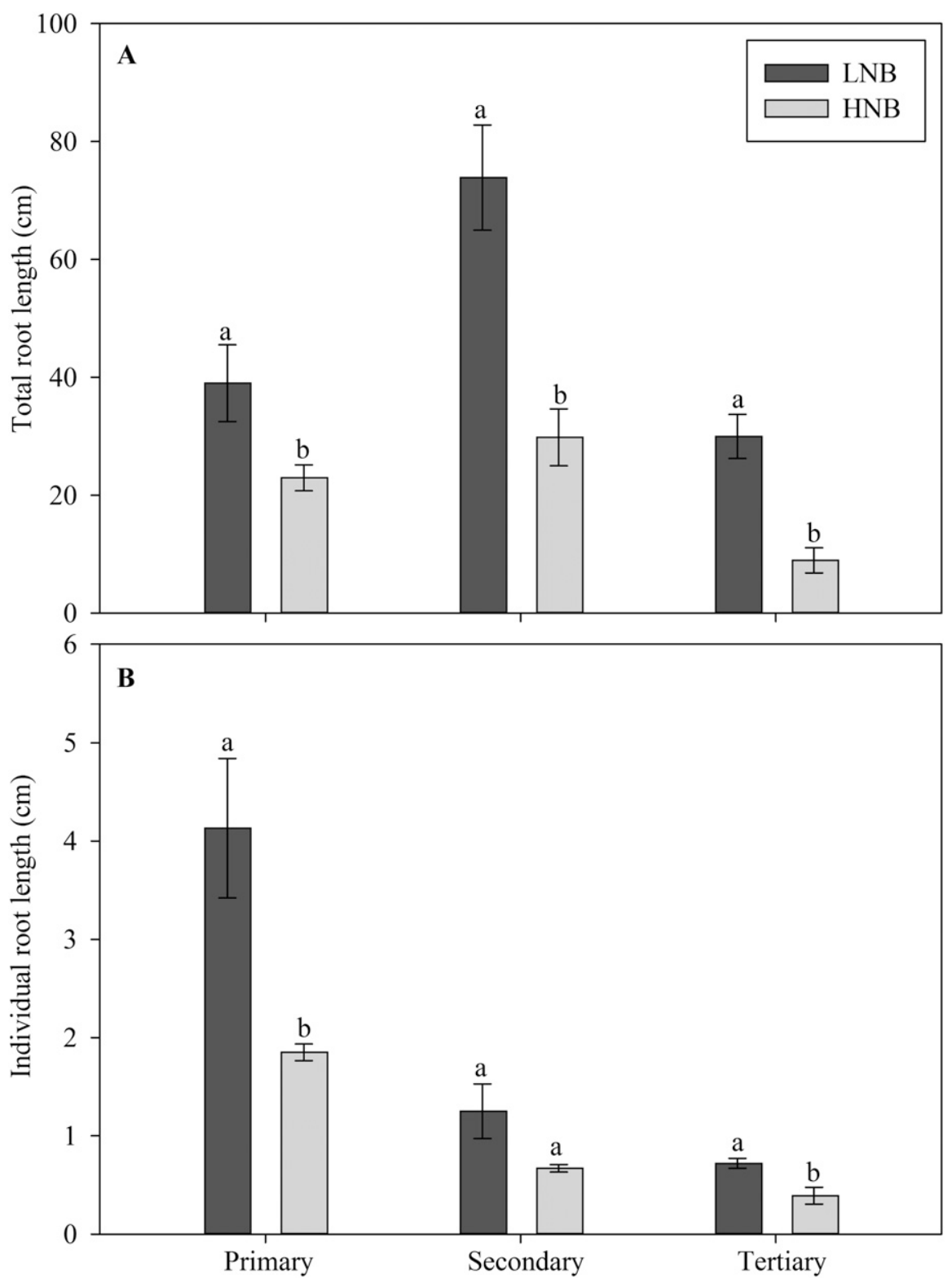

Fig. 7. Root length of primary, secondary, and tertiary roots of large crabgrass grown in split-pattern rhizobox with field soil amended with either $2 \%$ low-nutrient biochar (LNB) or $2 \%$ high-nutrient biochar (HNB). Total root length (A) represents the sum of all roots within a root order (primary, secondary, or tertiary). Individual root length (B) represents the average length of a single root within a root order (primary, secondary, or tertiary). Columns represent means; error bars represent standard error of the mean. Means within a root order with different letters indicate significant statistical difference $(P<0.05)$.

\section{Literature Cited}

Abiven, S., A. Hund, V. Martinsen, and G. Cornelissen. 2015. Biochar amendment increases maize root surface areas and branching: a shovelomics study in Zambia. Plant and Soil 395:45-55.

Biederman, L.A. and W.S. Harpole. 2013. Biochar and its effects on plant productivity and nutrient cycling: A meta-analysis. Glob. Change Biol. Bioenergy 5:202-214.
Brennan, A., E.M. Jimenez, M. Puschenreiter, J.A. Alburquerque, and C. Switzer. 2014. Effects of biochar amendment on root traits and contaminant availability of maize plants in a copper and arsenic impacted soil. Plant Soil 379:351360 .

Drew, M.C. 1975. Comparison of effects of a localized supply of phosphate, nitrate, ammonium and potassium on growth of seminal root system, and shoot, in barley. New Phytol. 75:479-490.

Hoveland, C.S., G.A. Buchanan, and M.C. Harris. 1976. Response of weeds to soil phosphorus and potassium. Weed Sci. 24:194-201.

Jeffery, S., F.G.A. Verheijen, M. van der Velde, and A.C. Bastos. 2011. A quantitative review of the effects of biochar application to soils on crop productivity using meta-analysis. Agr. Ecosyst. Environ. 144:175-187.

Khan, N. and S. Shea. 2012. Turf root enhancement by amendment of Jandakot sands of western Australia with different rates of biochar. J. Biobased Mater. Bioenergy 6:715723.

Lobet, G., L. Pages, and X. Draye. 2011. A novel image-analysis toolbox enabling quantitative analysis of root system architecture. Plant Physiol. 157:29-39.

Lynch, J.P. 2013. Steep, cheap and deep: An ideotype to optimize water and $\mathrm{N}$ acquisition by maize root systems. Ann. Bot. 112:347357.

Major, J., C. Steiner, A. Ditommaso, N.P.S. Falcao, and J. Lehmann. 2005. Weed composition and cover after three years of soil fertility management in the central Brazilian Amazon: Compost, fertilizer, manure and charcoal applications. Weed Biol. Mgt. 5:69-76.

Mitich, L.W. 1988. Crabgrass. Weed Technol 2:114-115.

Olmo, M., R. Villar, P. Salazar, and J.A. Alburquerque 2016. Changes in soil nutrient availability explain biochar's impact on wheat root development. Plant Soil 399:333-343.

Prendergast-Miller, M.T., M. Duvall, and S.P. Sohi. 2011. Localisation of nitrate in the rhizosphere of biochar-amended soils. Soil Biol. Biochem. 43:2243-2246.

Quilliam, R.S., K.A. Marsden, C. Gertler, J. Rousk, T.H. DeLuca, and D.L. Jones. 2012. Nutrient dynamics, microbial growth and weed emergence in biochar amended soil are influenced by time since application and reapplication rate. Agr. Ecosyst. Environ. 158:192199.

Schneider, C.A., W.S. Rasband, and K.W. Eliceiri. 2012. NIH image to ImageJ: 25 years of image analysis. Nat. Methods 9:671-675.

Smith, R.G. and D.A. Cox. 2014. Effects of soil amendments on the abundance of a parasitic weed, Yellow Rattle (Rhinanthus minor) in hay fields. Weed Sci. 62:118-124.

Soni, N., R.G. Leon, J.E. Erickson, J.A. Ferrell, M.L. Silveira, and M.C. Giurcanu. 2014. Vinasse and biochar effects on germination and growth of palmer amaranth (Amaranthus palmeri), sicklepod (Senna obtusifolia), and southern crabgrass (Digitaria ciliaris). Weed Technol. 28:694-702.

Spokas, K.A., J.M. Baker, and D.C. Reicosky. 2010. Ethylene: Potential key for biochar amendment impacts. Plant Soil 333:443-452.

Turner, F.A., K.S. Jordan, and R.C. Van Acker. 2012. Review: The recruitment biology and ecology of large and small crabgrass in turfgrass: Implications for management in the context of a cosmetic pesticide ban. Can. J. Plant Sci. 5:829-845. 